\title{
RELACIÓN ENTRE LAS EMISIONES DE CO, EL CONSUMO DE ENERGÍA Y EL PIB: EL CASO DE LOS CIVETS*
}

\author{
Jacobo Campo Robledo** \\ Wilmer Olivares $^{* * *}$
}

Recibido: enero 31 de 2012 • Aceptado: mayo 31 de 2013

\section{RESUMEN}

Este trabajo evalúa la relación que existe entre las emisiones de CO2 (dióxido de carbono), el consumo de energía y el PIB, para el grupo de países conocido como los CIVETS (Colombia, Indonesia, Vietnam, Egipto, Turquía y Sudáfrica), en el período 1985-2007. Para comprobar dichas relaciones se utiliza la metodología de datos de panel no estacionarios, acompañada de pruebas de raíces unitarias y de cointegración. Se concluye que, en el largo plazo, el crecimiento económico y el consumo de energía son determinantes del calentamiento global a través del incremento en las emisiones de CO2 para los CIVETS. Finalmente, se presenta evidencia empírica que comprueba la existencia de una Curva de Kuznets Ambiental para el grupo de países estudiado, que permite demostrar que los incrementos en el PIB per cápita incrementan las emisiones de $\mathrm{CO} 2$, pero a partir de determinado nivel de PIB las emisiones disminuyen.

\section{PALABRAS CLAVE}

Economía ambiental, emisiones de CO2, cointegración en panel, CIVETS.

\section{CLASIFICACIÓN JEL}

C33, Q50, Q56.

\section{CONTENIDO}

Introducción; 1. Revisión bibliográfica; 2. Metodología y datos; 3. Resultados; 4. Conclusiones; Bibliografía

Este artículo de es producto del proyecto denominado "Análisis empírico con bases de datos de los sectores público y privado" del grupo de investigación Finanzas y Política Económica, reconocido por Colciencias y que pertenece a la Facultad de Economía de la Universidad Católica de Colombia. Este proyecto fue financiado en su totalidad por la Universidad Católica de Colombia y ejecutado entre enero de 2011 y noviembre de 2011. Durante la ejecución de este proyecto, Wilmer Olivares se desempeñaba como asistente de investigación.

* Economista y Negociador Internacional, Universidad ICESI, Cali, Colombia. Magíster en Economía, Universidad del Rosario, Bogotá, Colombia. Líder del Grupo de Investigación de Finanzas y Política Económica. Docente-Investigador, Facultad de Economía, Universidad Católica de Colombia. Correo electrónico: jacampo@ucatolica.edu.co, jacobo.campo@gmail.com.

${ }^{* * *}$ Economista, Universidad Católica de Colombia, Bogotá, Colombia. Estudiante de la Especialización en Formulación y Evaluación Social y Económica de Proyectos. Asistente de Investigación, Facultad de Economía, Universidad Católica de Colombia. Correo electrónico: waolivares55@ucatolica.edu.co. 


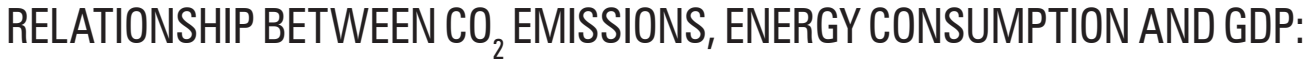 THE CASE OF THE CIVETS}

\section{ABSTRACT}

This paper evaluates the existent relation between $\mathrm{CO} 2$ emissions (Carbon Dioxide), energy consumption and GDP for the group of countries known as CIVETS (Colombia, Indonesia, Vietnam, Egypt, Turkey and South Africa), in the period 1985-2007. To prove these relations, a stationary data panel methodology is used supported by unitary root and cointegration tests. It is concluded that in the long term, economic growth and energy consumption are critical in explaining global warming through increases in $\mathrm{CO} 2$ emissions for CIVETS. Finally, empiric evidence is presented that proves the existence of a Kuznets Environmental Curve for the group of countries studied, which can prove that increases in the per capita GDP increase the $\mathrm{CO} 2$ emissions, but after the GDP reaches a certain level emissions decrease.

\section{KEY WORDS}

Environmental economy, CO2 emissions, cointegration panel, CIVETS,

\section{JEL CALSSIFICATION}

C33, Q50, 056

\section{CONTENT}

Introduction; 1. Bibliographical review; 2. Methodology and data; 3. Results; 4. Conclusions; Bibliography.

\section{RELAÇÃO ENTRE AS EMIÇÕES DE CO ${ }_{2}$ O CONSUMO DE ENERGIA E 0 PIB: $O$ CASO DOS CIVETS}

\section{RESUMO}

Este trabalho avalia a relação que existe entre as emissões de CO2 (Dióxido de Carbono), o consumo de energia e o PIB, para o grupo de países conhecido como os CIVETS (Colômbia, Indonésia, Vietnam, Egito, Turquia y Sul África), no período 1985-2007. Para comprovar estas relações se utiliza a metodologia de dados de painel não estacionários, acompanhada de provas de raízes unitárias e de cointegração. Conclui-se que, ao longo prazo, o crescimento económico e o consumo de energia são determinantes do aquecimento global por meio do incremento nas emissões de CO2 para os CIVETS. Finalmente, apresenta-se evidencia empírica que comprova a existência de uma Curva de Kuznets Ambiental para o grupo de países estudado, que permite demostrar que os incrementos no PIB per capita incrementam as emissões de CO2, mas a partir de determinado nível de PIB as emissões diminuem.

\section{PALAVRAS CHAVE}

Economia ambiental, emissões de CO2, cointegração em painel, CIVETS.

\section{CLASIFICAÇÃO JEL}

C33, Q50, Q56.

\section{CONTEUDO}

Introdução; 1. Revisão bibliográfica; 2. Metodologia e dados; 3. Resultados; 4. Conclusões; Bibliografia. 
Relación entre las emisiones de $\mathrm{CO}_{2}$, el consumo de energía y el PIB: el caso de los CIVETS

\section{INTRODUCCIÓN}

Al estudiar la relación existente entre las emisiones de $\mathrm{CO}_{2}$ (Dióxido de carbono), el consumo de energía y el producto interno bruto (PIB), para las economías que están llamadas a ser potencias mundiales (debido a su crecimiento anual y su importante atractivo para la inversión) como son los países que conforman el grupo de los CIVETS (Colombia, Indonesia, Vietnam, Egipto, Turquía y Sudáfrica), se debe tener en cuenta que este grupo de seis países es el grupo con las mayores expectativas de crecimiento durante las próximas dos décadas, con un crecimiento económico promedio de alrededor del $4 \%$ durante los siguientes veinte años; adicionalmente, las economías que hacen parte de los CIVETS son destinos potenciales para los inversionistas internacionales. Las estadísticas muestran que los CIVETS, incluso, pueden superar a otros grupos países como el G7 (Alemania, Canadá, Estados Unidos, Francia, Italia, Japón y Reino Unido) y los BRICS (Brazil, Rusia, India y China) que hoy en día son las economías más fuertes. Para los CIVETS esta perspectiva va aún más allá; las características propias de las economías en desarrollo limitan algunos aspectos de competencia, igualdad y comparabilidad; para este caso puntual los niveles de inflación y de deuda pública son muy similares para las seis economías; la situación de los países de la Eurozona y Estados Unidos, con la eventual crisis de la deuda, han presentado cifras alarmantes de la deuda pública de estos países, motivo por el cual la incertidumbre en los mercados se ha apoderado de los inversionistas llevándolos a buscar refugios en algunas economías como el grupo de los CIVETS o en las monedas más fuertes del mundo como el yen en Japón o el franco suizo en Suiza. No obstante, cuando las economías se esfuerzan por controlar las variables macroeconómicas y mantener la estabilidad financiera, descuidan las implicaciones que tienen estas políticas sobre el cambio climático.

Las emisiones de $\mathrm{CO}_{2}$ son consideradas las principales responsables del calentamiento global, y por tal razón su regulación es un tema muy importante para los gobiernos. Por ejemplo, el protocolo de Kyoto en 1997 se fijó como objetivo reducir los gases de efecto invernadero. Como los CIVETS es un grupo de países que han experimentado recientemente un rápido crecimiento económico, es importante estudiar el efecto que tiene el PIB sobre las emisiones de $\mathrm{CO}_{2}$. Adicionalmente, el amplio estudio que ha tenido la relación entre el consumo de energía y el PIB indica que los patrones de consumo de energía también inciden de manera positiva sobre las emisiones de $\mathrm{CO}_{2}$. En este sentido, si la relación es positiva, se deben proponer mecanismos en pro de la conservación y uso eficiente de la energía.

El objetivo de este trabajo es estudiar la relación que existe entre el consumo de energía, el PIB y las emisiones de $\mathrm{CO}_{2}$. A través de un modelo de datos panel 
macroeconómico no estacionario, se estiman las elasticidades de largo plazo de las emisiones de $\mathrm{CO}_{2}$ al consumo de energía y al PIB. Lo anterior, con el propósito de aportar evidencia empírica acerca de la relación que existe entre las emisiones de $\mathrm{CO}_{2}$ y algunos de sus determinantes, en este caso particular, el consumo de energía y el PIB. En adición, se presenta evidencia sobre la hipótesis de la existencia de una Curva de Kuznets Ambiental (CKA) para el grupo de países CIVETS.

De igual forma, la contribución de este trabajo a la literatura existente radica en que se emplean metodologías de datos panel no estacionarios, con pruebas de raíces unitarias para datos panel y pruebas de cointegración en panel, y se presenta evidencia empírica para un grupo de países (región económica) poco estudiado como el caso de los CIVETS.

El crecimiento de las economías esta determinado, en parte, por los hogares y las firmas, ya que a través de la producción de bienes y servicios por parte de las firmas se permite el consumo de bienes y servicios a los hogares, para satisfacer sus necesidades. Ahora bien, si el consumo de los hogares aumenta, las firmas aumentarán su producción para cubrir la demanda de los hogares, y el problema aquí es que dicho aumento productivo generará posibles externalidades negativas y positivas sumadas a posibles excesos frente al uso de los recursos ambientales, a través de incrementos en el consumo de energía e ingreso. Referida a este contexto, la construcción de políticas económicas, en la mayoría de los casos, no vincula los factores de contaminación y de degradación del medioambiente, para tratar de disminuir el impacto negativo de estos en la economía o limitar considerablemente el uso de estos recursos. He aquí entonces la importancia de conocer los efectos que tienen los incrementos del consumo de energía y del PIB sobre las emisiones de $\mathrm{CO}_{2}$ de una economía.

Este documento está dividido en cinco secciones, incluyendo la introducción, y organizado de la siguiente manera. En la primera sección se presenta una breve revisión de literatura empírica internacional sobre la relación entre las emisiones de $\mathrm{CO}_{2}$, el consumo de energía y el PIB. En la segunda, se exponen la metodología y los datos. En la tercera sección, se presentan los resultados de las pruebas de raíces unitarias y cointegración en panel, además de las estimaciones. Finalmente, en la cuarta y última sección se exponen las conclusiones.

\section{REVISIÓN BIBLIOGRÁFICA}

La relación entre el consumo de energía y el PIB ha sido estudiada por numerosos investigadores a lo largo de los últimos treinta años, 1980-2010; sin embargo, se hace necesario incluir en esta relación las emisiones de $\mathrm{CO}_{2}$, dada la creciente preocu- 
pación ambiental, debido el problema de cambio climático que se presenta en el ámbito mundial. A continuación se revisan algunos de los trabajos más relevantes en este tema, durante la década primera década del siglo XXI.

Hettige, Mani y Wheeler (2000) analizan datos internacionales y ponen a prueba la CKA. Miden el efecto del crecimiento económico a través de tres factores determinantes de la contaminación ambiental: un primer factor es la participación de la industria en la producción nacional; en segundo lugar la participación de los sectores contaminantes de la producción industrial y, por último, la intensidad de la contaminación por unidad de producto de estos sectores contaminantes. Los resultados muestran que la participación de la producción de la industria en la producción nacional sigue la trayectoria de la hipótesis de Kuznets, pero no los otros dos determinantes.

Por su parte, Dinda (2004) encuentra que la presión ambiental aumenta más rápido que los ingresos en las primeras etapas de desarrollo y reduce la velocidad en relación con el crecimiento del PIB en los niveles de ingresos más altos; además el autor revisa los desarrollos teóricos y empíricos que indagan sobre el fenómeno de la CKA.

Mehrara (2007) examina la causalidad que puede existir entre el consumo de energía per cápita y el PIB per cápita en un modelo panel de once países exportadores de petróleo, mediante el uso de pruebas de raíces unitarias en panel y pruebas de cointegración en panel. Los resultados muestran una causalidad unidireccional y un fuerte crecimiento del consumo de energía para los países exportadores de petróleo. En la misma vía, Soytas y Sari (2007) investigan la relación a largo plazo y encuentran una relación de causalidad en el sentido de Granger entre el crecimiento económico, las emisiones de dióxido de carbono y el consumo de energía en Turquía. El resultado más interesante es que las emisiones de carbono son causa del consumo de energía, pero no en sentido contrario. La falta de un vínculo a largo plazo de causalidad entre el ingreso y las emisiones implica que para reducir las emisiones de carbono, Turquía no tiene que renunciar al crecimiento económico.

Soytas, Sari y Ewing (2007), analizan el efecto del consumo de energía y las emisiones de carbono en los Estados Unidos, y evidencian una relación de causalidad de Granger entre el ingreso, el consumo de energía y las emisiones de carbono, además, con la fuerza laboral y la formación bruta de capital fijo. A través de la prueba de causalidad de Granger, encuentran que los ingresos y las emisiones de carbono causan en el largo plazo el uso de la energía. Según sus conclusiones, el crecimiento del ingreso por sí mismo no puede llegar a ser una solución a los pro- 
blemas ambientales. En otro trabajo Soytas y Sari (2009) refuerzan estos resultados para el caso de Estados Unidos.

Ang (2007a) examina la relación de largo plazo entre el PIB, las emisiones contaminantes y el consumo de energía en Malasia durante el período 1971 1999. En este estudio comprueba que la contaminación y el consumo de energía se relacionan de manera positiva con la producción en el largo plazo; además, muestra que existe una gran causalidad entre el crecimiento económico y el consumo energético, tanto en el corto como en largo plazo. En otro estudio similar, Ang (2007b) hace referencia a las relaciones dinámicas de causalidad entre las emisiones contaminantes y el consumo de energía. Sostiene que estas variables están muy relacionadas entre sí y, por lo tanto, su relación debe examinarse con un marco integrado. Los resultados proporcionan evidencia de la existencia de una relación de largo plazo bastante fuerte entre estas variables para el período 1960-2000. Por otra parte, los resultados apoyan el argumento de causalidad y comprueban que el crecimiento económico ejerce una influencia sobre el incremento del consumo de energía y la contaminación en el largo plazo.

Apergis y Payne (2009) examinan y demuestran que a largo plazo el consumo de energía de equilibrio tiene un impacto positivo y estadísticamente significativo en las emisiones, mientras que la producción real muestra la forma de U invertida, patrón asociado con la CKA y la dinámica de corto plazo que indica causalidad unidireccional entre el consumo de energía y el PIB real.

Belloumi (2009) aplica la prueba de cointegración propuesta por Johansen (1988, 1991) y utiliza sus resultados para examinar la relación causal entre el consumo de energía per cápita y PIB per cápita en Túnez, para el período 1971-2004. Los resultados indican que el PIB per cápita y el consumo de energía per cápita de Túnez están relacionados por un vector de cointegración en largo plazo; además, existe una relación de causalidad bidireccional entre las dos series y una causalidad unidireccional a corto plazo del consumo de energía al PIB.

Halicioglu (2009) examina de forma empírica las relaciones de causalidad entre las emisiones de carbono, el consumo de energía, el ingreso y el comercio exterior en el caso de Turquía, con datos de series de tiempo para el período 1960-2005. Los resultados indican que en el largo plazo existen dos formas de relación entre las variables. En la primera, las emisiones de carbono son determinadas por el consumo de energía, el ingreso y el comercio exterior. En la segunda, el ingreso está determinado por las emisiones de carbono, el consumo de energía y comercio exterior. En un siguiente estudio, Halicioglu (2011) estudia las relaciones causales entre la dinámica de la producción agregada, el consumo de energía, las exporta- 
ciones, el capital y el trabajo para Turquía, con datos de series de tiempo, para el período 1968-2008. Los resultados indican que existe una relación de largo plazo entre las variables. Adicionalmente, realiza un análisis de causalidad de Granger y concluye que en el largo plazo, la causalidad se comporta de forma interactiva a través del término de corrección de errores, del trabajo, el capital, las exportaciones y el consumo de energía a la producción agregada.

Luzzati y Orsini (2009) estudian la relación entre el consumo de energía y el PIB per cápita de 113 países durante el período 1971-2004. Dado que la energía ha sido poco explorada en la CKA los autores la toman como indicador de la presión del medioambiente en general.

Pao (2009) examina la causalidad de Granger entre el consumo de energía eléctrica y el crecimiento económico de Taiwán para el período 1980-2007, mediante el uso de un modelo de corrección de errores. Los resultados indican que el consumo de energía eléctrica y el PIB real están cointegrados, y que no hay causalidad unidireccional a corto y largo plazo. Por su parte, Zhang y Cheng (2009) analizan la existencia y la dirección de causalidad de Granger entre crecimiento económico, el consumo de energía y las emisiones de carbono en China, para el período 1960 2007, y sugieren una causalidad en el sentido de Granger unidireccional entre el PIB y el consumo de energía, y una causalidad de Granger unidireccional entre el consumo de energía y las emisiones de carbono en el largo plazo. Demuestran que ni las emisiones de carbono ni el consumo de energía inciden en el crecimiento económico en China, durante el período de estudio.

Pao y Tsai (2010) examinan la dinámica de las relaciones causales entre las emisiones de $\mathrm{CO}_{2}$, el consumo de energía, el producto interno Bruto y la inversión extranjera directa para el grupo de países del BRIC (Brasil, Rusia, India y Corea), durante el período 1971-2005, a excepción de Rusia (1990-2005). Los resultados indican que a largo plazo el consumo de energía de equilibrio tiene un impacto positivo y estadísticamente significativo en las emisiones, mientras que el PIB real muestra la forma de U invertida, patrón asociado con la CKA.

Gómez (2010) estudia el crecimiento económico, consumo de energía y emisiones contaminantes en la economía mexicana. Para esto utiliza una metodología de ciclos económicos reales, vectores autorregresivos (modelos VAR) y la prueba de causalidad de Granger, y obtiene como resultado que existe una relación positiva entre crecimiento económico, el consumo de energía y las emisiones de $\mathrm{CO}_{2}$, que el consumo de energía antecede al crecimiento económico, y que tanto cambios en el consumo de energía como crecimiento económico se explican por incrementos en el consumo de energía de los sectores de la economía industrial y de transporte. 


\section{Curva de Kuznets ambiental}

La hipótesis de la CKA fue introducida por Panayotou (1993), al estudiar el efecto que tiene el crecimiento económico en ciertos indicadores de aire y tierra. La hipótesis, en sí, sostiene que el incremento del PIB aumenta la contaminación hasta cierto nivel de ingreso per cápita, a partir del cual comienza a reducirse la contaminación. La existencia de esta relación de U invertida, como se muestra en la figura 1, ha sido fundamentada por múltiples factores o efectos, entre otros, el efecto composición, desplazamiento, el ambiente como un bien de lujo, el progreso tecnológico y las regulaciones ambientales. Todos estos, argumentados por numerosos autores como Hettige, Mani y Wheeler (1998), Grossman y Krueger (1991), Low y Yeats (1992) y Rothman (1998).

Figura 1. Curva de Kuznets Ambiental

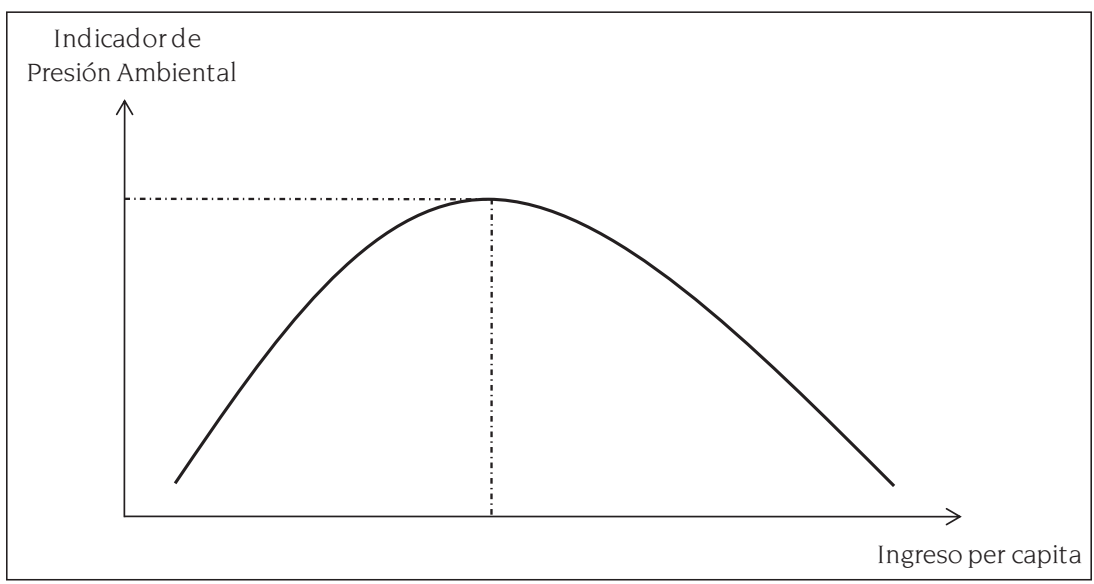

Fuente: elaborado por los autores.

\section{METODOLOGÍA Y DATOS}

En esta sección se expone la metodología empleada para determinar los efectos que tienen el PIB y el consumo de energía sobre las emisiones de $\mathrm{CO}_{2}$. En adición, se presentan las gráficas de los datos con los cuales se aplica dicha metodología.

\subsection{Metodología: datos panel}

Durante las últimas dos décadas, 1990-2010, los datos panel han sido utilizados como herramienta de análisis por parte de investigadores de diversas áreas para estudiar las relaciones entre diferentes variables. La razón principal es que esta metodología combina una dimensión de tiempo (series de tiempo) con otra transversal (corte transversal). 
Al trabajar con datos de panel macroeconómicos, los cuales contemplan una serie de tiempo mayor a la cantidad de individuos, se debe tener en cuenta la existencia de una relación de largo plazo entre las variables que se analizan para el grupo de individuos. En otras, palabras, se debe asegurar que exista una relación de cointegración para evitar el problema de obtener resultados espurios como lo sostienen Engle y Granger (1987). Entorf (1997), Kao (1999) y Phillips y Moon (1999), quienes introdujeron el término de relaciones espurias en el uso de datos panel, cuando las observaciones de tiempo son mayores al número de individuos en un panel. Para esto es necesario aplicar pruebas de raíces unitarias que permiten determinar el orden de integración de las series de tiempo, y pruebas de cointegración para determinar si las series de tiempo de cada país están o no cointegradas, y con esto evitar los resultados espurios.

La implementación de esta metodología de datos de panel vincula el hecho de que los individuos, firmas, bancos o países como en este caso (los CIVETS) son heterogéneos. Los análisis de series de tiempo y de corte transversal no tratan de controlar esta heterogeneidad con el riesgo de obtener resultados sesgados. Una ventaja adicional es que permiten estudiar de una mejor manera la dinámica de los procesos de ajuste de los datos tomados para los países CIVETS.

Según la literatura internacional sobre economía energética, se puede plantear la siguiente ecuación con el fin de determinar la relación de largo plazo entre las emisiones de $\mathrm{CO}_{2}$, el consumo de energía y el PIB. Esta forma funcional ha sido empleada por otros autores como Pao y Tsai (2010), Apergis y Payne (2009) y Halicioglu (2009):

$$
\mathrm{LCO}_{i t}=\beta_{0}+\beta_{1} \mathrm{LCE}_{i t}+\beta_{2} \mathrm{LPIB}_{i t}+\beta_{3} \mathrm{LPIB}_{i t}^{2}+\varepsilon_{i t}
$$

Donde $\left(\mathrm{LCO}_{i t}\right)$ es el logaritmo de las emisiones de $\mathrm{CO}_{2}$ de cada país (i) en el período $(t),\left(\mathrm{LCE}_{i t}\right)$ es el logaritmo del consumo de energía de cada país (i) en el período $(t)$ y $\left(\right.$ LPIB $\left._{i t}\right)$ es el logaritmo del PIB de cada país (i) en el periodo $(t)$. Se emplea la variable $\left(\mathrm{LPIB}^{2}{ }_{i t}\right)$ con el propósito de probar la hipótesis de la existencia de una Curva de Kuznets Ambiental; por tanto, si existe dicha curva, el signo que se espera tenga el coeficiente que acompaña a la variable $\left(\mathrm{LPIB}_{i t}\right)$ sea positivo, mientras que el signo del coeficiente que acompaña a la variable ( $\mathrm{LPIB}_{\text {it }}^{2}$ ) será negativo. Por otro lado, se espera que el coeficiente que acompaña a la variable $\left(\mathrm{LCE}_{i t}\right)$ tenga signo positivo.

\subsubsection{Pruebas de raíces unitarias para el panel}

Se considera primero el orden de integración de las series emisiones de $\mathrm{CO}_{2}$, consumo de energía y PIB, expresadas en términos per cápita. Se emplean las pruebas para 
datos panel desarrolladas por Im, Pesaran y Shin (2003) conocida como la prueba IPS, Levin, Lin y Chu (2002), Breitung (2000), Maddala y Wu (1999) (Fisher tipo ADF) y Choi (2001) (Fisher tipo PP). Las pruebas de Maddala y Wu (1999) e Im, Pesaran y Shin (2003) permiten la heterogeneidad entre los individuos de los datos del panel. Las pruebas de raíces unitarias para panel tienen su fundamento en las pruebas desarrolladas para series de tiempo, pero tienen una ventaja sobre estas últimas, y es que al combinar series de tiempo y datos de sección cruzada se obtienen más grados de libertad lo cual mejora las propiedades de los estimadores, y además corrigen la heterogeneidad no observada.

\subsubsection{Prueba de cointegración para el panel}

Luego de comprobar que las series son integradas de orden uno, es decir, que contienen una raíz unitaria en el panel, se continúa con la prueba de cointegración, con el fin de encontrar evidencia sobre la existencia de una relación entre las variables en el largo plazo, y de esta forma evitar la presencia de relaciones espurias. Lo anterior se prueba a través de la conocida prueba de cointegración en panel heterogéneo de Pedroni (2000, 2004). Esta prueba se basa en siete estadísticos divididos en dos grupos; el primero está basado en el estadístico de Phillips y Ouliaris (1990), el cual está definido como:

$$
\tilde{Z}_{p}=\sum_{i=1}^{\mathrm{N}} \frac{\sum_{t=1}^{\mathrm{T}}\left(\hat{\varepsilon}_{i t-1} \Delta \hat{\varepsilon}_{i t}-\hat{\lambda}_{i}\right)}{\left(\sum_{t=1}^{\mathrm{T}} \hat{\varepsilon}_{i t-1}^{2}\right)}
$$

En donde $\left(\hat{\varepsilon}_{i t}\right)$ es estimado de la ecuación (1), $\left(\lambda_{i}=\frac{1}{2}\left(\hat{\sigma}_{i}^{2}-\hat{s}_{i}^{2}\right)\right)$, para lo cual $\left(\hat{\sigma}_{i}^{2}\right)$ es la varianza de largo plazo de $\left(\hat{\varepsilon}_{i t}\right)$ y $\left(\hat{s}_{i}^{2}\right)$ es la varianza contemporánea. El segundo grupo de estadísticos se basan en la razón de varianza, definida de la matriz de varianzas y covarianzas de largo plazo. El método de estimación es Fully Modified Ordinary Least Squares (FMOLS), ya que este estimador corrige los problemas de endogeneidad y heterogeneidad que están presentes en las pruebas de cointegración basadas en los errores del modelo y estimadas por OLS. Una mayor descripción de esto puede encontrarse en Pedroni (2000).

\subsection{Datos}

A continuación se presentan las variables utilizadas en este documento. El PIB per cápita medido (en dólares a precios constantes de 2000), el consumo de energía eléctrica (kWh por habitante), las emisiones de $\mathrm{CO}_{2}$ (toneladas métricas per cápita). 
Todas las series fueron obtenidas del Banco Mundial. Los gráficos 1 al 3 muestran el comportamiento de cada una de las series de los países CIVETS.

Gráfico 1. Emisiones de $\mathrm{CO}_{2}$ (toneladas de dióxido de carbono per cápita), 1985-2007

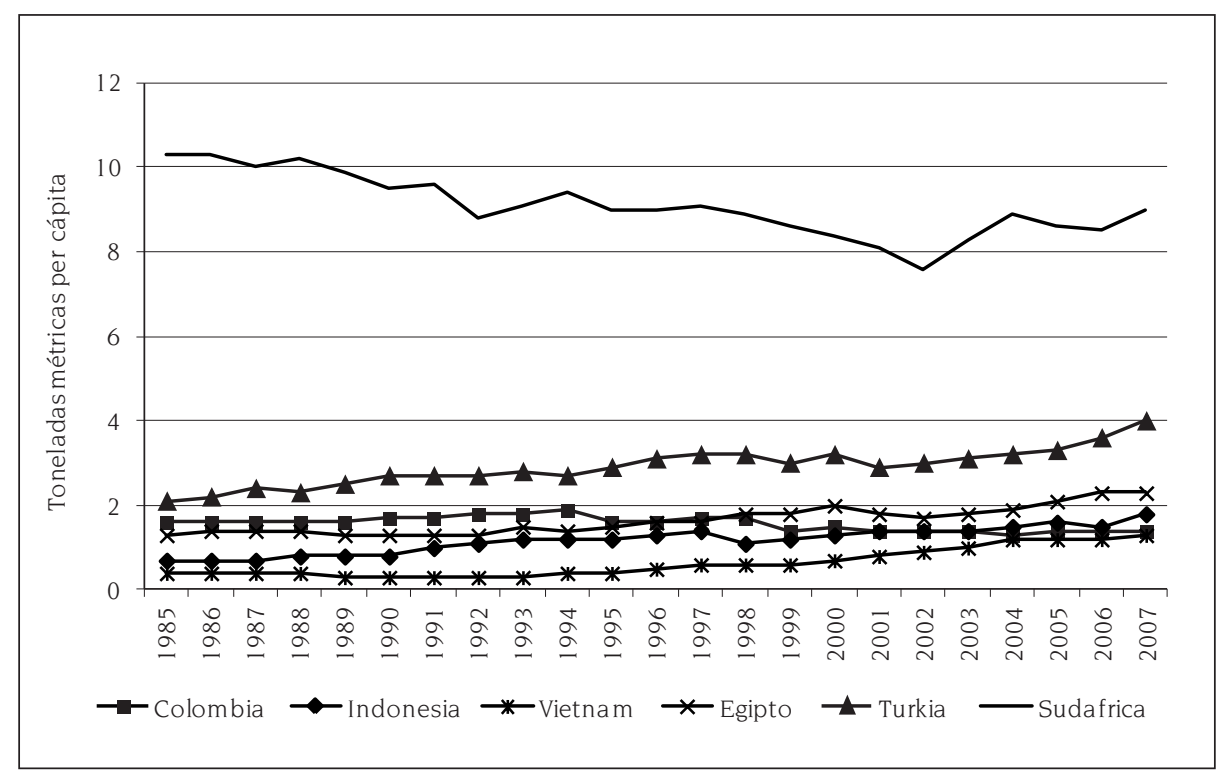

Fuente: elaboración de los autores con base en información del Banco Mundial

En los gráficos 1 y 2 se evidencia que Sudáfrica es el país que tiene más emisiones de $\mathrm{CO}_{2}$ y consumo de energía per cápita, en comparación con los demás países CIVETS. En el caso colombiano se observa que su comportamiento está dentro del rango de los demás países del grupo, y por ejemplo, Indonesia es el país con más crecimiento del grupo y una participación porcentual en emisiones de $\mathrm{CO}_{2}$ de un $4,8 \%$. Egipto e Indonesia son los países más homogéneos con una participación baja en emisiones de $\mathrm{CO}_{2}$ por persona.

El consumo de energía de los CIVETS se ve reflejado en el gráfico 2 donde sobresale el consumo de energía de Sudáfrica a diferencia de los otros países del grupo, los cuales presentan un comportamiento homogéneo, que puede ser a causa de los hábitos en el consumo, como por ejemplo el mínimo uso de combustibles fósiles o una alta tasa de crecimiento económico durante la última década. 
Gráfico 2. Consumo de energía per cápita,

1985-2007

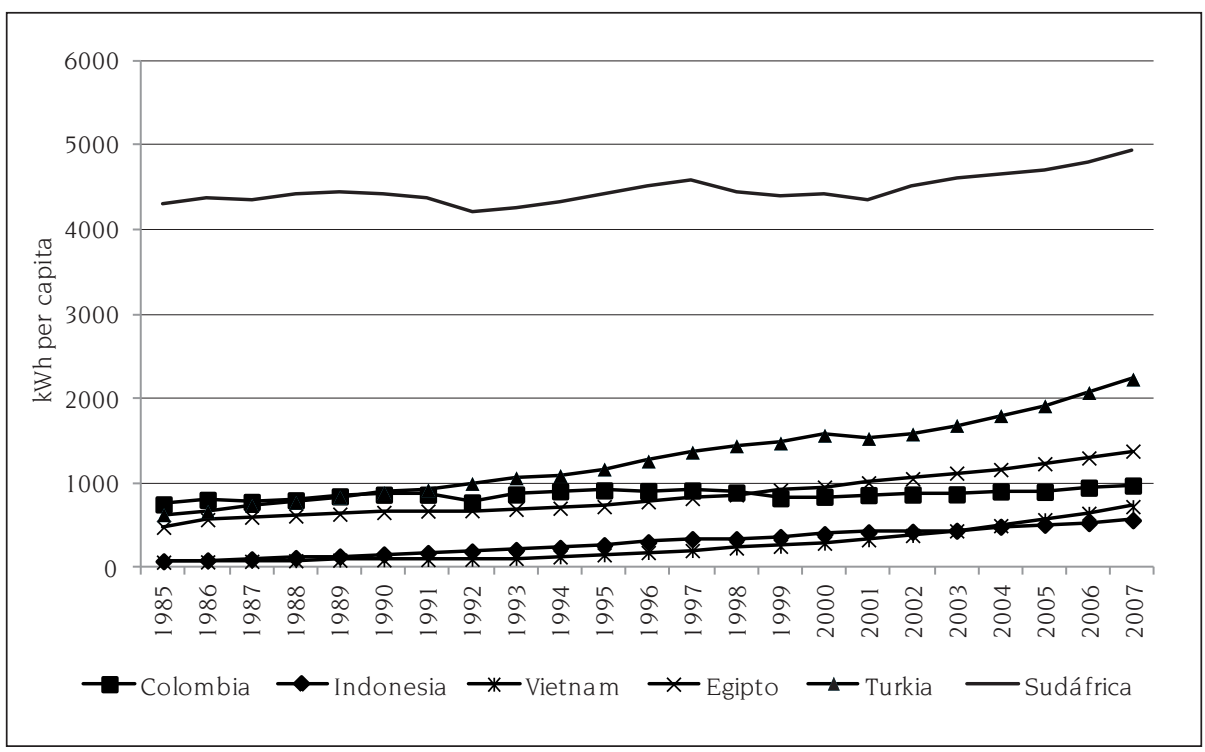

Fuente: elaboración de los autores con base en información del Banco Mundial

Gráfico 3. PIB real per cápita,

$$
\text { 1985-2007 }
$$

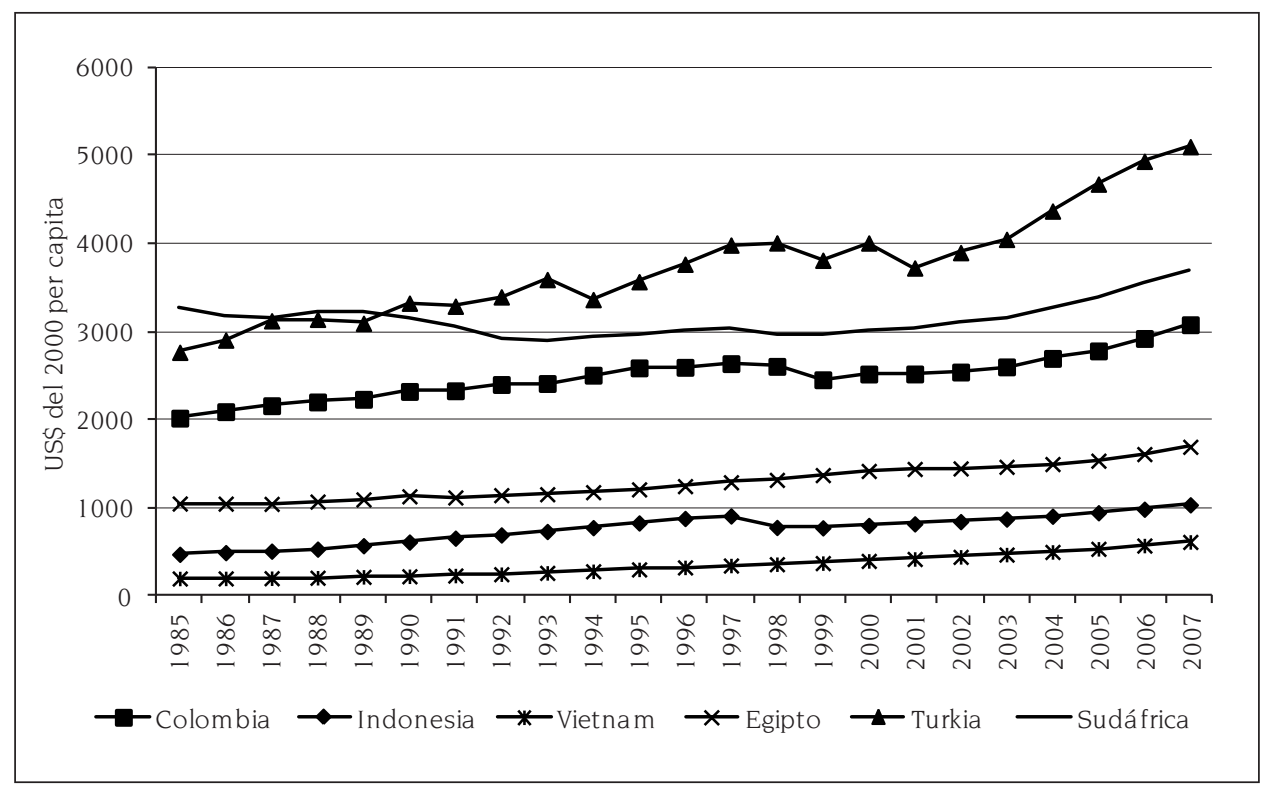

Fuente: elaboración de los autores con base en información del Banco Mundial 
Relación entre las emisiones de $\mathrm{CO}_{2}$, el consumo de energía y el PIB: el caso de los CIVETS

El gráfico 3 permite inferir que Turquía es el país que ha presentado un mayor crecimiento económico por persona a lo largo de la serie con un aumento promedio de $2,40 \%$.

En las tablas 1, 2 y 3, se presentan estadísticas descriptivas discriminadas por quinquenios, ya que las serie de tiempo al parecer no son estacionarias. En la tabla 1 se observa que el país con mayor PIB per cápita del grupo es Turquía durante los últimos tres quinquenios, además de ser el que presenta mayor desviación, lo que permite ver que en términos de PIB per cápita es el más sólido del grupo. Colombia se ubica en el segundo puesto, en términos de desviación del PIB per cápita.

Tabla 1. Estadísticas descriptivas del PIB per cápita por quinquenios (US\$)

\begin{tabular}{|l|c|c|c|c|c|c|c|c|}
\cline { 2 - 9 } \multicolumn{1}{c|}{} & \multicolumn{2}{c|}{$1986-1990$} & \multicolumn{2}{c|}{$1991-1995$} & \multicolumn{2}{c|}{$1996-2000$} & \multicolumn{2}{c|}{$2001-2005$} \\
\cline { 2 - 9 } \multicolumn{1}{c|}{} & Media & Desviación & Media & Desviación & Media & Desviación & Media & Desviación \\
\hline Colombia & 2205,58 & 85,18 & 2451,16 & 101,28 & 2566,44 & 74,46 & 2632,10 & 109,75 \\
\hline Indonesia & 546,48 & 48,44 & 740,50 & 67,95 & 831,34 & 62,06 & 880,66 & 49,83 \\
\hline Vietnam & 213,14 & 10,32 & 268,10 & 27,39 & 363,82 & 27,83 & 477,28 & 45,40 \\
\hline Egipto & 1082,00 & 36,01 & 1164,80 & 34,79 & 1333,00 & 67,71 & 1481,42 & 38,86 \\
\hline Turquía & 3121,62 & 149,02 & 3445,60 & 131,94 & 3919,00 & 117,08 & 4146,80 & 380,89 \\
\hline Sudáfrica & 3189,92 & 33,48 & 2956,48 & 59,27 & 3003,32 & 27,58 & 3193,78 & 140,40 \\
\hline
\end{tabular}

Fuente: Banco Mundial, cálculos propios

Tabla 2. Estadísticas descriptivas del consumo de energía por quinquenios $(\mathrm{kWh})$

\begin{tabular}{|l|c|c|c|c|c|c|c|c|}
\cline { 2 - 9 } \multicolumn{1}{c|}{} & \multicolumn{2}{c|}{$1986-1990$} & \multicolumn{2}{c|}{$1991-1995$} & \multicolumn{2}{c|}{$1996-2000$} & \multicolumn{2}{c|}{$2001-2005$} \\
\cline { 2 - 9 } \multicolumn{1}{c|}{} & Media & Desviación & Media & Desviación & Media & Desviación & Media & Desviación \\
\hline Colombia & 822,20 & 35,43 & 869,18 & 56,20 & 879,16 & 41,06 & 883,38 & 19,88 \\
\hline Indonesia & 123,06 & 27,94 & 220,36 & 36,42 & 352,66 & 35,44 & 459,70 & 39,02 \\
\hline Vietnam & 86,54 & 10,00 & 122,28 & 23,06 & 236,52 & 43,28 & 447,36 & 93,70 \\
\hline Egipto & 618,02 & 32,61 & 697,26 & 21,67 & 871,68 & 70,62 & 1118,48 & 88,80 \\
\hline Turquía & 784,64 & 86,92 & 1053,46 & 93,28 & 1428,42 & 115,02 & 1707,68 & 157,73 \\
\hline Sudáfrica & 4408,64 & 41,16 & 4321,08 & 93,29 & 4474,58 & 78,49 & 4573,56 & 132,85 \\
\hline
\end{tabular}

Fuente: Banco Mundial 
Tabla 3. Estadísticas descriptivas de emisiones de $\mathrm{CO}_{2}$ por quinquenios (toneladas métricas per cápita)

\begin{tabular}{|l|c|c|c|c|c|c|c|c|}
\cline { 2 - 9 } \multicolumn{1}{c|}{} & \multicolumn{2}{c|}{$1986-1990$} & \multicolumn{2}{c|}{$1991-1995$} & \multicolumn{2}{c|}{$1996-2000$} & \multicolumn{2}{c|}{ 2001-2005 } \\
\cline { 2 - 9 } \multicolumn{1}{c|}{} & Media & Desviación & Media & Desviación & Media & Desviación & Media & Desviación \\
\hline Colombia & 1,62 & 0,04 & 1,76 & 0,11 & 1,58 & 0,13 & 1,38 & 0,04 \\
\hline Indonesia & 0,76 & 0,05 & 1,14 & 0,09 & 1,26 & 0,11 & 1,46 & 0,09 \\
\hline Vietnam & 0,36 & 0,05 & 0,34 & 0,05 & 0,60 & 0,07 & 1,02 & 0,18 \\
\hline Egipto & 1,36 & 0,05 & 1,40 & 0,10 & 1,76 & 0,17 & 1,86 & 0,15 \\
\hline Turquía & 2,42 & 0,19 & 2,76 & 0,09 & 3,14 & 0,09 & 3,10 & 0,16 \\
\hline Sudáfrica & 9,98 & 0,31 & 9,18 & 0,32 & 8,80 & 0,29 & 8,30 & 0,49 \\
\hline
\end{tabular}

Fuente: Banco Mundial

En la tabla 2 se evidencia que Sudáfrica es el país del grupo con mayor consumo de energía per cápita (en promedio). Sin embargo, la mayor desviación la presenta Turquía. Vietnam, por su parte, es el país con menores niveles de consumo de energía. En la tabla 3 se puede observar que el país que más emisiones de $\mathrm{CO}_{2}$ per cápita produce es Sudáfrica, mientras que Vietnam presenta el menor número de emisiones de $\mathrm{CO}_{2}$.

\section{RESULTADOS}

En esta sección se presentan los resultados de las pruebas de raíces unitarias y cointegración en datos panel. La tabla 4 expone los resultados de las cinco pruebas de raíces unitarias presentadas en la sección anterior, y se indica para cada una de las variables en niveles, el estadístico y la probabilidad. Se puede observar que para ninguna de las pruebas se puede rechazar la hipótesis nula de raíz unitaria, con lo cual se concluye que las series no son estacionarias en niveles.

Tabla 4. Raíz unitaria para variables en niveles

\begin{tabular}{|l|c|c|c|c|c|c|}
\hline \multirow{2}{*}{ Prueba } & \multicolumn{2}{|c|}{ Emisiones de $\mathrm{CO}_{2}$} & \multicolumn{2}{c|}{ Consumo de energía } & \multicolumn{2}{c|}{ PIB } \\
\cline { 2 - 7 } & Estadístico & Probabilidad & Estadístico & Probabilidad & Estadístico & Probabilidad \\
\hline $\begin{array}{l}\text { Im, Pesaran and Shin } \\
\text { (Estadístico W) }\end{array}$ & 0,36 & 0,64 & 0,57 & 0,72 & 1,47 & 0,93 \\
\hline ADF - Fisher (Chi-cuadrado) & 4,87 & 0,77 & 2,04 & 0,73 & 6,08 & 0,91 \\
\hline PP - Fisher (Chi-cuadrado) & 5,34 & 0,72 & 3,49 & 0,48 & 11,41 & 0,49 \\
\hline Levin, Lin E Chu (t) & $-0,63$ & 0,26 & $-0,84$ & 0,20 & 1,42 & 0,92 \\
\hline
\end{tabular}


Relación entre las emisiones de $\mathrm{CO}_{2}$, el consumo de energía y el PIB: el caso de los CIVETS

\begin{tabular}{|c|c|c|c|c|c|c|}
\hline \multirow{2}{*}{ Prueba } & \multicolumn{2}{|c|}{ Emisiones de $\mathrm{CO}_{2}$} & \multicolumn{2}{c|}{ Consumo de energía } & \multicolumn{2}{c|}{ PIB } \\
\cline { 2 - 7 } & Estadístico & Probabilidad & Estadístico & Probabilidad & Estadístico & Probabilidad \\
\hline Breitung (Estadístico t) & 0,73 & 0,77 & 2,02 & 0,98 & 3,47 & 1,00 \\
\hline
\end{tabular}

${ }^{(* * *)}$ Indica el rechazo de la hipótesis nula al $1 \%$ de significancia

(*) Indica el rechazo de la hipótesis nula al $5 \%$ de significancia

(*) Indica el rechazo de la hipótesis nula al $10 \%$ de significancia

Fuente: elaboración de los autores con base en los resultados de las estimaciones

A continuación se presenta el resultado de las pruebas de raíces unitarias aplicadas a las series en primeras diferencias, con el fin de descartar la presencia de más de una raíz unitaria. La tabla 5 expone dichos resultados para cada variable, y se presentan el estadístico y la probabilidad. Se concluye que las series en diferencias son estacionarias, ya que se puede rechazar la hipótesis nula de que existe raíz unitaria

Tabla 5. Raíz unitaria para variables en primera diferencia

\begin{tabular}{|l|c|c|c|c|c|c|}
\hline \multirow{2}{*}{ Prueba } & \multicolumn{2}{|c|}{$\Delta\left(\right.$ Emisiones de $\left.\mathrm{CO}_{2}\right)$} & \multicolumn{2}{c|}{$\begin{array}{c}\text { (Consumo de } \\
\text { energía })\end{array}$} & \multicolumn{2}{c|}{$\Delta$ PIB } \\
\cline { 2 - 7 } & Estadístico & Probabilidad & Estadístico & Probabilidad & Estadístico & Probabilidad \\
\hline $\begin{array}{l}\text { Im, Pesaran and Shin } \\
\text { (Estadístico W) }\end{array}$ & $-4,82$ & $0,00^{* * *}$ & $-2,66$ & $0,00^{* * *}$ & $-2,19$ & $0,01^{* *}$ \\
\hline ADF - Fisher (Chi-cuadrado) & 45,82 & $0,00^{* * *}$ & 26,85 & $0,00^{* * *}$ & 23,07 & $0,03^{* *}$ \\
\hline PP - Fisher (Chi-cuadrado) & 87,30 & $0,00^{* * *}$ & 61,95 & $0,00^{* * *}$ & 41,01 & $0,00^{* * *}$ \\
\hline Levin, Lin E Chu (t) & $-4,79$ & $0,00^{* * *}$ & $-1,30$ & 0,096 & $-3,73$ & $0,00^{* * *}$ \\
\hline Breitung (Estadístico t) & $-4,51$ & $0,00^{* * *}$ & $-2,39$ & $0,00^{* * *}$ & $-2,04$ & $0,02^{* *}$ \\
\hline
\end{tabular}

(*) Indica el rechazo de la hipótesis nula al $10 \%$ de significancia

(**) Indica el rechazo de la hipótesis nula al $5 \%$ de significancia

${ }^{(* * *)}$ Indica el rechazo de la hipótesis nula al 1 \% de significancia

Fuente: elaboración de los autores con base en los resultados de las estimaciones

El resultado de la prueba de cointegración de Pedroni $(2000,2004)$ para los CIVETS, expuesta en la sección anterior, se presenta en la tabla 6. Se puede observar que cinco de los siete estadísticos de esta prueba permiten rechazar la hipótesis nula de no cointegración al $5 \%$ de significancia; por lo tanto, existe evidencia estadística fuerte a favor de una relación de cointegración entre las variables emisiones de $\mathrm{CO}_{2}$ per capita, el consumo de energía per cápita y el PIB per cápita. 
Tabla 6. Pruebas de cointegración

\begin{tabular}{|c|c|c|}
\hline & \multirow[b]{2}{*}{ Estadístico } & \multirow[b]{2}{*}{ Probabilidad } \\
\hline & & \\
\hline \multicolumn{3}{|l|}{ Within-dimension } \\
\hline Panel v-Statistic & 1,95 & $0,02^{* *}$ \\
\hline Panel rho-Statistic & $-0,72$ & 0,24 \\
\hline Panel PP-Statistic & $-2,53$ & $0,00^{* *}$ \\
\hline Panel ADF-Statistic & $-2,07$ & $0,02^{* *}$ \\
\hline \multicolumn{3}{|l|}{ Between-dimension } \\
\hline Group rho-Statistic & 0,82 & \\
\hline Group PP-Statistic & $-1,70$ & $0,04^{* *}$ \\
\hline Group ADF-Statistic & $-2,79$ & $0,00^{*}$ \\
\hline
\end{tabular}

${ }^{(* *)}$ Indica el rechazo de la hipótesis nula al $5 \%$ de significancia

Fuente: elaboración de los autores con base en los resultados de las estimaciones

En la tabla 7 se observan los resultados de la estimación de la relación de largo plazo con base en la prueba de cointegración de Pedroni (1999, 2004), para la ecuación (1), y se observa que todos los coeficientes son estadísticamente significativos al $1 \%$, y, además, tienen el signo esperado a-priori.

Tabla 7. Relación de largo plazo, ecuación de cointegración

\begin{tabular}{|l|c|c|c|}
\hline \multicolumn{1}{|c|}{ Variable } & Coeficiente & Estadístico $t$ & Probabilidad \\
\hline Constante & $-8,10$ & $-3,70$ & 0,00 \\
LCE & 0,42 & 5,82 & 0,00 \\
LPIB & 1,52 & 2,78 & 0,01 \\
(LPIB)A2 & $-0,10$ & $-2,94$ & 0,00 \\
\hline R-Cuadrado & 0,84 & & \\
R-Cuadrado Ajustado & 0,84 & & \\
Estadístico F & 238,72 & & \\
Probabilidad (F) & 0,00 &
\end{tabular}

Fuente: elaboración de los autores con base en los resultados de las estimaciones

En forma resumida, se puede expresar la ecuación de cointegración de la siguiente manera (errores estándar):

$$
\mathrm{LCO}_{i t}=\underset{(2,19)}{-8,10}+\underset{(0,07)}{0,42} \mathrm{LCE}_{i t}+\underset{(0,54)}{1,52 \mathrm{LPIB}_{i t}}-\underset{(0,03)}{0,09 \mathrm{LPIB}_{i t}^{2}}
$$

Los anteriores resultados muestran que las emisiones de $\mathrm{CO}_{2}$ son inelásticas (menor a uno) a cambios en el consumo de energía, es decir, un incremento en 
$1 \%$ del consumo de energía genera en el largo plazo un incremento en 0,41 \% de las emisiones de $\mathrm{CO}_{2}$ del panel de datos de los CIVETS. Por otro lado, la elasticidad panel de las emisiones de $\mathrm{CO}_{2}$ con respecto al PIB en el largo plazo se formula como sigue:

$$
\begin{gathered}
1,52 \mathrm{LPIB}_{i t}-0,09 \mathrm{LPIB}_{i t}^{2} \\
\frac{\partial \mathrm{LCO}_{i t}}{\partial \mathrm{LPIB}_{i t}}=1,52-(2 \times 0,09) \mathrm{LPIB}_{i t}=0 \\
\frac{\partial \mathrm{LCO} 2_{i t}}{\partial \mathrm{LPIB}_{i t}}=1,52-0,19 \mathrm{LPIB}_{i t}=0 \\
\mathrm{LPIB}_{i t}=\frac{1,52}{0,19}=7,99
\end{gathered}
$$

Lo anterior implica que el punto de inflexión se encuentra cuando el PIB alcanza un nivel de 7,99 en logaritmos (o 2.951,29 en millones de dólares del 2000 per cápita). Estos resultados refuerzan la hipótesis de la existencia de una CKA, ya que el nivel de emisiones de $\mathrm{CO}_{2}$ primero se incrementa con el PIB, después se estabiliza y finalmente decrece. La elasticidad del PIB sobre las emisiones de $\mathrm{CO}_{2}$ será mayor a 1, cuando el PIB per cápita es menor a 2,73' (en logaritmos). En resumen, en el largo plazo, las emisiones de $\mathrm{CO}_{2}$ son inelásticas al consumo de energía y elásticas al PIB si este es inferior a 2,73, mientras que inelástica si el PIB es mayor a 2,73.

En resumen, los resultados sugieren una respuesta considerable de las emisiones de $\mathrm{CO}_{2}$ ante cambios en el PIB, y una respuesta relativamente baja de las emisiones de $\mathrm{CO}_{2}$ ante incrementos en el consumo de energía. Resultados similares encontraron Arpergis y Payne (2009) y Pao y Tsai (2011), para un grupo de países diferente.

\section{CONCLUSIONES}

Durante la década pasada, las regiones y países en el ámbito mundial se han concienciado sobre el efecto negativo que pueden tener los patrones de consumo de energía, el crecimiento del PIB, entre otras, sobre el medioambiente. En otras palabras, se han dado cuenta de que lo más importante, aún por encima del crecimiento económico, es el impacto negativo que los patrones de consumo mundial pueden generar sobre el medioambiente.

En este trabajo se presenta una primera aproximación al estudio de los determinantes de las emisiones de $\mathrm{CO}_{2}$, para los CIVETS (Colombia, Indonesia, Vietnam, Egipto, Turquía, Sudáfrica) del período 1985-2007. Con datos panel, se estima la

Este valor se obtiene $\left(=^{(1,52-1 /} / 0,19\right)$. 
relación emisiones de $\mathrm{CO}_{2}$, PIB real, y consumo de energía, con el fin de conocer los coeficientes de esta relación en el largo plazo y poder analizar el impacto, además de contribuir al cumplimiento de la hipótesis de la existencia de una CKA.

Los resultados que se presentan en el documento implican que existe una relación de largo plazo entre las emisiones de $\mathrm{CO}_{2}$, el consumo de energía y el PIB. En adición, se encuentra que la elasticidad de las emisiones de $\mathrm{CO}_{2}$ con respecto al consumo de energía es menor a uno (inelástica), y la elasticidad de las emisiones de $\mathrm{CO}_{2}$ con respecto al PIB sugiere la existencia de una Curva de Kuznets Ambiental, ya que tiene forma de U invertida con punto de inflexión en un nivel de PIB per cápita de 7,99 (en logaritmos).

De igual forma, los resultados sugieren que existe una relación de causalidad del PIB a las emisiones de $\mathrm{CO}_{2}$, y del consumo de energía a las emisiones de $\mathrm{CO}_{2}$, lo cual implica que en el largo plazo el crecimiento económico es un determinante del cambio climático a través de las emisiones de $\mathrm{CO}_{2}$, al menos para la región conformada por los países estudiados, conocida como los CIVETS. En concreto, en el equilibrio, un incremento en $1 \%$ del consumo de energía genera en el largo plazo un incremento en $0,41 \%$ de las emisiones de $\mathrm{CO}_{2}$ del panel de datos de los CIVETS. Por otro lado, las emisiones de $\mathrm{CO}_{2}$ son elásticas al PIB si este es inferior a 2,73 (en logaritmos), mientras que inelástica si el PIB es mayor a 2,73 (en logaritmos).

Un resultado importante es que el consumo de energía tiene un efecto positivo sobre las emisiones de $\mathrm{CO}_{2}$, pero relativamente bajo, como se mencionó anteriormente, lo cual sugiere que las políticas encaminadas a promover el uso eficiente y la conservación de la energía disminuyen las emisiones de $\mathrm{CO}_{2}$ y, por tanto, estas pueden contribuir a reducir el calentamiento global. Por otro lado, la evidencia encontrada a favor de la Curva de Kuznets Ambiental indica que si bien el crecimiento económico incrementa el nivel de emisiones de $\mathrm{CO}_{2}$, este incremento se estabiliza para luego presentar una reducción de las emisiones. Esta reducción evidencia que los países se vuelven más eficientes en la producción, con mejores procesos productivos y cada vez menos contaminantes.

No obstante, quedan en la agenda de investigación varios aspectos por estudiar, tales como cuál es el efecto que tiene la profundización financiera o flujos de capitales de esta región de los CIVETS sobre las emisiones de $\mathrm{CO}_{2}$, ya que como sostienen Frankel y Romer (1999) la profundización financiera y el desarrollo pueden atraer inversión extranjera directa y mayores inversiones en investigación y desarrollo, lo cual acelera el crecimiento económico de las economías y afecta la dinámica del desempeño ambiental. 
Relación entre las emisiones de $\mathrm{CO}_{2}$, el consumo de energía y el PIB: el caso de los CIVETS

\section{BIBLIOGRAFÍA}

Ang, J. (2007a). Economic development, pollutant emissions and energy consumption in Malaysia. En: Journal of Policy Modeling, Vol. 30, pp. 271-278.

Ang, J. (2007b). $\mathrm{CO}_{2}$ emissions, energy consumption, and output in France. En: Energy Policy, Vol. 35, pp. 4772-4778.

Apergis, N. y Payne, J. (2009). CO2 emissions, energy usage, and output in Central America. En: Energy Policy, Vol. 37, pp. 3282-3286.

Belloumi, M. (2009). Energy consumption and GDP in Tunisia: cointegration and causality analysis. En: Energy Policy, Vol. 37, pp. 2745-2753.

Breitung, J. (2000). The local power of some unit root tests for panel data. En: Advances in Econometrics, Vol. 15, pp. 161-177.

Choi, I. (2001). Unit root test for panel data. En: Journal of International Money y Finance, Vol 20, pp. 249-272.

Dinda, S. (2004). "Environmental Kuznets Curve Hypothesis: A Survey". Ecological Economics, Vol. 49, pp. 431-455.

Engle, R.F. y Granger, C. (1987). Co-integration and Error- Correction: Representation, estimation and testing. En: Econometrica, Vol. 55, N. ${ }^{\circ}$ 2, pp. 251-276.

Entorf, H. (1997). Random walks with drifts: Nonsense regression and spurious fixed-effect estimation. En: Journal of Econometrics, Vol. 80, pp. 287-296.

Frankel, J. y Romer, D. (1999). Does trade cause growth? En: American Economic Review, Vol. 89, pp. 379-399.

Gómez, C. (2010). Crecimiento económico, consumo de energía y emisiones contaminantes en la economía mexicana. En: Revista Fuente, Vol. 3, N. ${ }^{\circ}$ 9, pp. 67-80.

Grossman, G. y Krueger, A. (1991). Environmental impacts of a North American Free Trade Agreement. En: Nacional Bureau of Economic Research. Working Paper N. ${ }^{\circ} 3914$.

Halicioglu, F. (2011). A dynamic econometric study of income, energy and exports in Turkey. En: Energy, Vol. 36, pp. 3348-3354.

Halicioglu, F. (2009). An econometric study of CO2 emissions, energy consumption, income and foreign trade in Turkey. En: Energy Policy, Vol. 37, pp. 1156-1164.

Hettige, H.; Mani, M. y Wheeler, D. (1998). Industrial pollution in economic development. The world Bank Development Research Group. Policy Research Working Paper 1876. 
Hettige, H.; Mani, M. y Wheeler, D. (2000). Industrial pollution in economic development: the environmental Kuznets curve revisited. En: Journal of Development Economics, Vol. 62, pp. 445-476.

Im, K.; Pesaran, M. y Shin, Y. (2003). Testing for unit roots in heterogeneous panels. En: Journal of Econometrics, Vol. 115, pp. 53-74.

Johansen, S. (1988). Statistical Analysis of Cointegration Vectors. En: Journal of Economic Dynamics and Control, Vol. 12, N. ${ }^{\circ} 2-3$, pp. 231-254.

Johansen, S. (1991). Estimation and Hypothesis Testing of Cointegration Vectors in Gaussian Vector Autoregressive Models. En: Econométrica, Vol. 59, N. 6, pp. 1551-1580.

Kao, C. (1999). Spurious regression and residual-based test for cointegration in panel data. En: Journal of Econometrics, Vol. 90, pp. 1 44.

Levin, A.; Lin, C. y Chu, J. (2002). Unit root in panel data: Asymptotic and finite simple Properties. En: Journal of Econometrics, Vol. 108, N..$^{\circ}$, pp. 1-24.

Low, P. y Yeats, A. (1992). Do "dirty" industries migrate? En: P. Low (Ed.), International trade and the environment. Washington, D. C., World Bank.

Luzzati, T. y Orsini, M. (2009). Investigating the energy-environmental Kuznets curve. En: Energy, Vol. 34, pp. 291-300.

Maddala, G. y Wu, S. (1999). A comparative study of unit root test with panel data and a new simple test. En: Oxford Bulletin of Economics y Statistics, Vol. 61, pp. 631-652.

Mehrara, M. (2007). Energy consumption and economic growth: The case of oil exporting countries. En: Energy Policy, Vol. 35, pp. 2939-2945.

Panayotou, T. (1993). Empirical tests and policy analysis of environmental degradation at different stages of economic development. Geneva, International Labor Office, Technology and Employment Programme.

Pao, H. (2009). Forecast of electricity consumption and economic growth in Taiwan by state space modelling. En: Energy, Vol. 34, pp. 1779-1791.

Pao, H. y Tsai, Ch. (2010). CO2 emissions, energy consumption and economic growth in BRIC countries. En: Energy Policy, Vol. 38, pp. 7850-7860.

Pao, H. y Tsai, C. (2011). Multivariate Granger causality between CO2 emissions, energy consumption, FDI (foreign direct investment) and GDP (gross domestic product): Evidence from a panel of BRIC (Brazil, Russian Federation, India, and China) countries. En: Energy, Vol. 36, pp. 685-693.

Pedroni, P. (1999). Critical Values for Cointegration Tests in Heterogeneous Panels with Multiple Regressors. En: Oxford Bulletin of Economics and Statistics, Vol. 61, pp. 653-670. 
Relación entre las emisiones de $\mathrm{CO}_{2}$, el consumo de energía y el PIB: el caso de los CIVETS

Pedroni, P. (2000). Fully modified OLS for heterogeneous cointegrated panels. En: Advances in Econometrics, Vol. 15, pp. 93-130.

Pedroni, P. (2004). Panel Cointegration: asymptotic y finite sample propierties of pooled time series with an application to the PPP hypothesis: New Results. En: Econometric Theory, Vol. 20, pp. 597-627.

Phillips, P. y Moon, H. (1999). Linear regression limits theory for nonstationary panel data. En: Econometrica, Vol. 67, pp. 1057-1111.

Phillips y Ouliaris (1990). Asymptotic Properties of Residual Based Test for Cointegration. En: Econometrica, Vol. 58, pp. 165-193.

Rothman, D. (1998). Environmental Kuznets curve-real progress or passing the buck. En: Ecological Economics, Vol. 25, N. ${ }^{\circ}$, pp. 177-194.

Soytas, U.; Sari, R. y Ewing, Bradley T. (2007). Energy consumption, income, and carbon emissions in the United States. En: Ecological Economics, Vol. 62, pp. 482-489.

Soytas, U. y Sari, R. (2007). The relationship between energy and production: Evidence from Turkish manufacturing industry. En: Energy Economics, Elsevier, Vol. 29, N. 6, pp. 1151-1165.

Soytas, U. y Sari, R. (2009). Energy consumption, economic growth, and carbon emissions: Challenges faced by an EU candidate member. En: Ecological Economics, Vol. 62, pp. 1667-1675.

Zhang, X. y Cheng, X. (2009).Energy consumption, carbon emissions, and economic growth in China. En: Ecological Economics, Vol. 68, pp. 2706-2712. 
